\title{
Traços de personalidade e suas alterações em mulheres com lúpus
}

\author{
Danusa Céspedes Guizzo Ayache ${ }^{1}$, Izaías Pereira da Costa ${ }^{2}$
}

\begin{abstract}
RESUMO
Objetivo: Avaliar traços de personalidade e suas alterações em pacientes do sexo feminino com Lúpus Eritematoso Sistêmico (LES), procurando relacionar mudanças na atividade da doença com alterações nos traços de personalidade dessas pacientes. Pacientes e Métodos: Foram selecionadas 20 pacientes do Ambulatório de Reumatologia do Hospital Universitário da Universidade Federal de Mato Grosso do Sul (UFMS). Após a avaliação inicial, elas foram reavaliadas em três e em seis meses, em relação à presença de Transtornos Psiquiátricos (particularmente alterações da personalidade) e atividade do lúpus. Além das avaliações clínicas psiquiátricas e reumatológicas, as escalas utilizadas para avaliação das pacientes foram a Escala Fatorial de Ajustamento Emocional/Neuroticismo (EFN) e a Systemic Lupus Erythematosus Disease Activity Index (SLEDAI). Resultados: Em todos os tempos analisados não houve relação significativa entre a pontuação na SLEDAI e o escore da EFN. Não foi encontrada, portanto, associação entre alterações da personalidade e atividade do lúpus. De acordo com a EFN, seis pacientes (30\%) apresentaram pontuação sugestiva de Transtornos de Personalidade, porém apenas duas (10\%) tiveram esse diagnóstico pela avaliação psiquiátrica. Também não foi encontrado um padrão típico de personalidade ou prevalência de um Transtorno de Personalidade específico na amostra; entretanto, houve uma prevalência importante de Depressão (65\%). Conclusões: Verificou-se na população estudada que as pacientes lúpicas podem apresentar os mais variados tipos de comportamento e sintomas psiquiátricos, não havendo nelas um padrão típico de personalidade. Não foi encontrada uma relação significativa entre as alterações de personalidade e atividade da doença.
\end{abstract}

Palavras-chave: transtornos psiquiátricos, alterações da personalidade, lúpus eritematoso sistêmico.

\section{INTRODUÇÃO}

O Lúpus Eritematoso Sistêmico (LES) é uma doença inflamatória crônica, multisistêmica, de causa desconhecida e natureza autoimune, caracterizada pela presença de diversos autoanticorpos. Evolui com diversas manifestações clínicas e com períodos de exacerbação e remissão. ${ }^{1}$ Tem prevalência maior em pacientes do sexo feminino, em idade fértil e com história familiar de doença autoimune. ${ }^{2,3}$

Há consenso entre a comunidade científica ${ }^{1,3,4}$ quanto à origem multifatorial da doença, envolvendo fatores hormo- nais, genéticos, ambientais, infecciosos (virais) e estresse psicológico, hoje considerado por muitos autores como de particular importância no desencadeamento da doença e de seus agravamentos. ${ }^{2,5}$

O Sistema Nervoso Central (SNC) é frequentemente atingido, gerando sintomas neurológicos e/ou psiquiátricos. ${ }^{1,3,6}$ Alguns trabalhos têm correlacionado essas manifestações clínicas à presença de alguns anticorpos, como o anti-P ribossomal, anti-SSa, anti-DNA e anti-fosfolipídios, entre outros. ${ }^{7,8,9}$ Especula-se ainda que a ativação do Sistema Imunológico

\footnotetext{
Recebido em 02/11/2008. Aprovado, após revisão, em 01/09/2009. Declaramos a inexistência de conflitos de interesse.

1. Médica psiquiatra; Professora assistente de Psiquiatria do Departamento de Clínica Médica da Faculdade de Medicina da Universidade Federal de Mato Grosso do Sul (UFMS); Coordenadora da residência médica em Psiquiatria da UFMS; Mestre em Ciências da Saúde pelo Programa Multi-institucional de PósGraduação em Ciências da Saúde (UNB-UFG-UFMS)

2. Médico Reumatologista; Professor Associado II do Departamento de Clínica Médica da Faculdade de Medicina da Universidade Federal de Mato Grosso do Sul (UFMS); Mestre e Doutor em Medicina pela Universidade de São Paulo (USP); Professor do Curso de Pós-Graduação em Ciências da Saúde-UFMS; Membro da Academia Brasileira de Reumatologia; Coordenador do Programa de Residência Médica em Reumatologia do NHU/UFMS

Endereço para correspondência: Danusa Céspedes Guizzo Ayache. Rua Rio Grande do Sul, 1590. Vila Célia - Campo Grande, MS - Brasil. E-mail: rdayache@ terra.com.br
} 
possa resultar em alterações em neurotransmissores e, consequentemente, em comportamentos. ${ }^{10}$

Em 1999, um subcomitê do American College of Rheumatology (ACR) classificou 19 síndromes neuropsiquiátricas relacionadas ao LES. Entre elas, foram classificadas como síndromes psiquiátricas: estado confusional agudo, distúrbios cognitivos, psicose, transtornos de humor e de ansiedade. ${ }^{1}$ Não foram lembradas, entretanto, as alterações da personalidade.

Ayache \& Costa, ${ }^{11}$ em revisão de literatura sobre alterações da personalidade em pacientes com LES, verificaram que vários autores concluíram que os fatores psicológicos (incluindo traços de personalidade) têm importância como codeterminantes, desencadeantes ou exacerbadores da doença. Alguns verificaram ainda que alterações da personalidade podem ser decorrentes do estresse psicológico imposto pelo LES, da atividade da doença no SNC e/ou do uso de medicações como imunossupressores e corticoides. ${ }^{12-28}$

Vertzman \& Pinheiro, apud Lemle, ${ }^{29}$ estão desenvolvendo na Universidade Federal do Rio de Janeiro (UFRJ) o projeto "Patologias Narcísicas e Doenças Autoimunes: Estudo Comparativo sob a óptica da psicanálise". Segundo esses autores, a partir de dados da experiência clínica e de algumas referências da literatura, os pacientes lúpicos teriam uma configuração psíquica narcísica (seriam pessoas egoístas, arrogantes, voltadas somente para si). Os autores estão fazendo um estudo comparativo, avaliando de forma qualitativa (de acordo com o referencial psicanalítico) pacientes lúpicos e melancólicos. Até o presente momento, afirmam ter encontrado entre os pacientes com LES os mais variados tipos de organização psíquica, e não uma maior incidência de personalidades narcísicas.

Nery et al. ${ }^{30}$ avaliaram 71 pacientes com LES quanto à presença e intensidade do transtorno depressivo, e em $23 \%$ deles foi diagnosticada depressão maior e tendência a uma maior gravidade do Lúpus. A gravidade da depressão foi diretamente correlacionada com a atividade da doença e a incapacidade funcional. Esses autores admitem a hipótese de que a depressão maior em pacientes com atividade da doença no SNC seja uma manifestação da doença, mediada por mecanismos autoimunes, e isso merece mais investigação.

Nery et $a l .{ }^{31}$ realizaram estudo para estimar a prevalência de transtornos psiquiátricos em pacientes com LES e sua associação com anticorpos anti-P. Selecionaram 71 pacientes com LES do sexo feminino sem manifestações neurológicas. Os transtornos psiquiátricos mais prevalentes nos últimos 30 dias foram os do humor (26,8\%) e de ansiedade (46,5\%), que também foram os mais prevalentes durante a vida (transtornos do humor $69 \%$ e de ansiedade 52,1\%). Indivíduos com e sem manifestações psiquiátricas não diferiram quanto aos parâmetros clínicos e laboratoriais, incluindo a presença ou ausência de anticorpos anti-P, atividade da doença e índice de dano acumulado. Os autores concluíram que os transtornos de humor e de ansiedade são os transtornos psiquiátricos mais frequentemente observados em pacientes com LES do sexo feminino sem manifestações neurológicas. As formas leve/ moderada desses transtornos não estão associadas a anticorpos anti-P em pacientes com LES.

A revisão de literatura, portanto, é inconclusiva quanto à presença de um padrão de personalidade nas pacientes lúpicas. Quanto às alterações de personalidade desencadeadas pela doença ou uso de medicação, os resultados dos trabalhos são ainda controversos. A avaliação da personalidade da paciente lúpica é bastante complexa, devido à variedade de fatores que podem causar interferência. Verificando a necessidade de mais estudos que se proponham a elucidar essas questões, decidimos realizar esta pesquisa, que teve como objetivos: a) avaliar traços de personalidade e suas possíveis implicações em pacientes do sexo feminino com o diagnóstico de LES, procurando relacionar mudanças na atividade da doença com alterações nos traços de personalidade; b) verificar se existe um ou mais padrões característicos de personalidade no grupo estudado; c) verificar a presença de padrões de comportamento ou transtornos psiquiátricos associados à doença, em suas fases ativa e inativa.

\section{PACIENTES E MÉTODOS}

\section{Amostra}

Os indivíduos desta pesquisa foram 20 pacientes atendidas no Ambulatório de Colagenoses do Serviço de Reumatologia do Hospital Universitário (HU) da Universidade Federal de Mato Grosso do Sul (UFMS). O projeto foi submetido ao Comitê de Ética da UFMS e aprovado em 27/04/2004 (No do protocolo: 380/2004).

Foram incluídas no estudo pacientes do sexo feminino com 18 a 50 anos de idade e diagnóstico de LES pelos critérios do ACR.$^{32}$ Foram excluídas: pacientes analfabetas; com pontuação no Miniexame do Estado Mental ${ }^{33}$ inferior ao esperado para sua idade e escolaridade; com quadros psiquiátricos graves ou em uso de psicotrópicos na primeira avaliação; com outras doenças crônicas associadas e com gravidez suspeita ou confirmada.

As pacientes foram selecionadas para a pesquisa de forma consecutiva, através da revisão dos prontuários do ambulatório, de maio a junho de 2004. Aquelas que pelos dados do prontuário encaixavam-se nos critérios acima expostos, foram convidadas a participar da pesquisa. Desse modo, selecio- 
namos 40 pacientes, porém nem todas foram localizadas ou concordaram em participar. Conseguimos uma amostra de 20 pacientes, que pode ser considerada não probabilística e por julgamento.

\section{Instrumentos de Avaliação}

Além da avaliação clínica psiquiátrica e reumatológica, foram utilizadas as seguintes escalas:

a) Escala Fatorial de Ajustamento Emocional/Neuroticis$\mathrm{mo}-\mathrm{EFN}^{34}$

Esta escala é um instrumento para avaliação de uma dimensão da personalidade humana denominada Neuroticismo/Ajustamento Emocional, também denominado Fator N. Segundo Hutz \& Nunes. ${ }^{34} \mathrm{O}$ fator $\mathrm{N}$ apresenta estreita correlação com os transtornos de personalidade catalogados nos sistemas diagnósticos psiquiátricos atuais, como a $4^{\mathrm{a}}$ edição do Diagnostic and Statistical Manual of Mental Disorders, o DSM-IV ${ }^{35}$ e a Classificação Internacional de Transtornos Mentais e de Comportamento, a CID-10. ${ }^{36}$

$\mathrm{O}$ instrumento consta de 82 itens, distribuídos nas seguintes subescalas: ${ }^{34}$

N1 (Vulnerabilidade - 23 itens): Escores altos neste fator sugerem um Transtorno de Personalidade Dependente; já escores muito baixos, um Transtorno de Personalidade Esquiva.

N2 (Desajuste Psicossocial - 14 itens): Escores altos neste fator sugerem Transtornos de Personalidade como o Antissocial e Borderline. Ainda não se tem clareza do significado de escores baixos para este fator.

N3 (Ansiedade - 25 itens): Escores altos neste fator sugerem Transtornos de Ansiedade; escores baixos, sintomas como impulsividade e comportamentos de risco.

N4 (Depressão - 20 itens): Escores altos sugerem Transtornos Depressivos; escores baixos, dificuldade para detectar e enfrentar problemas, o que pode se relacionar diretamente com as estratégias de enfrentamento de uma doença.

Para o cálculo geral da Escala, deve-se efetuar a soma da pontuação dos escores padronizados das quatro subescalas (N1, N2, N3 e N4) através de procedimentos específicos. Escores gerais entre 80 a 120 pontos são esperados para a maior parte da população. Valores mais altos ou mais baixos podem indicar um Transtorno de Personalidade, exigindo uma investigação mais aprofundada por parte do psiquiatra ou psicólogo. ${ }^{34}$

A partir do escore bruto de cada subescala, pode ser calculado um escore percentílico para cada fator. Um valor maior que 70 pontos, ou menor que 30 encontrado em algum desses escores pode indicar um distúrbio psicológico e/ou psiquiátrico mais específico. ${ }^{37}$ b) SLEDAI - Systemic Lupus Erythematosus Disease Activity Index ${ }^{38}$

A SLEDAI é uma escala utilizada internacionalmente para avaliar a atividade do lúpus, baseada na avaliação clínica (reumatológica) e em exames complementares padronizados. A pontuação na escala pode variar de 0 a 105 pontos (pts). Adotamos a classificação preconizada por Cook et al.:39 inatividade (0 pts); atividade leve (1 a 3 pts); atividade moderada (4 a 7 pts) e atividade severa (maior ou igual a 8 pts).

\section{Cronograma das avaliações e seguimento das pacientes}

As avaliações clínicas (reum-atológicas e psiquiátricas) e a aplicação das escalas foram realizadas juntamente com os exames complementares, no decorrer de uma semana, a fim de se comparar possíveis alterações na atividade do LES com alterações da personalidade. Após a avaliação inicial, as pacientes foram reavaliadas após três meses e seis meses, a fim de verificar possíveis alterações na EFN de acordo com o curso da doença e/ou fatores externos, como estressores psicossociais que poderiam emergir em momentos diferentes. Portanto, consideramos este um estudo de coorte prospectivo.

\section{Análise dos Dados}

Foi realizada estatística descritiva e analítica, com os seguintes testes: teste não paramétrico de Friedman, com pós-teste de Student-Newman-Keuls; teste do qui-quadrado; teste de McNemar e teste exato de Fisher. Os resultados foram apresentados na forma de estatística descritiva, gráficos e tabelas; e para tal foi utilizado o Software SigmaStat, versão 2.0, considerando relações ou diferenças significativas quando o valor de $\mathrm{P}$ foi menor que 0,05 . $^{40}$

\section{RESULTADOS}

\section{Dados Sociodemográficos}

A amostra foi composta principalmente por pacientes afrodescendentes $(50 \%)$ e brancas (45\%); a idade média foi de $31,75 \pm 8,30$ anos (média \pm desvio padrão da média). A maior parte das pacientes declarou-se casada $(60 \%)$ e realizando apenas atividades domésticas (55\%); a média de escolaridade foi de $10,1 \pm 2,0$ anos.

\section{Resultados da EFN e avaliação psiquiátrica}

A Tabela 1 nos mostra os escores gerais da EFN nas três avaliações: na avaliação inicial, 15\% das pacientes apresentaram 
escores anormais; na $2^{\mathrm{a}}$ avaliação, $26 \%$ anormais; na $3^{\mathrm{a}}$ avaliação, $35 \%$ anormais. Observamos que houve uma relação significativa entre a pontuação na EFN e os tempos do estudo. Esta relação foi observada entre a $1^{\mathrm{a}}$ e $2^{\mathrm{a}}$ avaliação (teste McNemar, $\mathrm{P}=0,019$ ) e entre a $1^{\mathrm{a}}$ e $3^{\mathrm{a}}$ avaliação $(\mathrm{P}=0,037)$. Entre a $2^{\mathrm{a}}$ e $3^{\mathrm{a}}$ avaliação, não houve relação significativa $(\mathrm{P}=0,118)$. Para este teste o escore pela EFN foi subdividido em: a) anormal (menor que 80 ou maior que 120 pontos); e b) normal (entre 80 e 120 pontos). Estes resultados juntos indicam que com o tempo houve uma diminuição da frequência de casos classificados pela EFN como normais e um aumento dos casos classificados como anormais, especialmente para escores menores que 80 pontos. Das pacientes com escores "anormais", apenas uma (5\%) recebeu um diagnóstico de Transtorno de Personalidade pela avaliação psiquiátrica.

Verificamos na Tabela 2 que não houve uma relação significativa entre a avaliação psiquiátrica e o escore obtido na EFN (teste do qui-quadrado, $\mathrm{P}=0,060$ ). Das três pacientes (15\%) classificadas como anormais pela EFN, apenas uma (5\%) teve um diagnóstico psiquiátrico pela avaliação clínica (Episódio Depressivo Moderado e Transtorno de Personalidade Dependente). Além disso, 14 pacientes (70\%) com diagnóstico psiquiátrico obtiveram um escore geral normal na EFN.

Observamos ainda que 15 pacientes $(75 \%)$ apresentavam um ou dois diagnósticos psiquiátricos em T0. O diagnóstico mais prevalente em nossa amostra, de acordo com a avaliação clínica, foi o de Episódio Depressivo Moderado (EDM), o único diagnóstico psiquiátrico em 10 pacientes (50\%); duas pacientes $(10 \%)$ apresentaram um diagnóstico de EDM e Deficiência Mental Leve; uma paciente (5\%), EDM e Transtorno de Personalidade Dependente. Também tivemos uma paciente (5\%) com diagnóstico de Transtorno Afetivo Bipolar e Transtorno de Personalidade Borderline; uma paciente (5\%) com diagnóstico de Uso Abusivo de Álcool e Dependência de Tabaco; somente cinco pacientes ( $25 \%$ da amostra) não tiveram nenhum diagnóstico psiquiátrico na avaliação inicial.

Na Tabela 3 verificamos o número de pacientes e pontuação dos escores percentílicos das subescalas da EFN, na avaliação inicial: não houve relação significativa entre os escores percentílicos e os fatores da EFN (teste do qui-quadrado, $\mathrm{P}=0,243$ ). Para este teste os escores pela EFN foram subdivididos em: a) anormal (menor que 30 pontos e maior que 70 pontos); e b) normal (entre 30 e 70 pontos).

Em relação ao fator N1, 40\% das pacientes apresentaram um valor acima do esperado, sugerindo um possível Transtorno de Personalidade Dependente; entretanto, tivemos apenas uma paciente $(5 \%)$ diagnosticada, pela avaliação psiquiátrica, com este transtorno, associado à EDM. Em relação ao fator N2, apenas uma paciente (5\%) apresentou um valor acima do espe- rado, sugerindo um Transtorno de Personalidade Antissocial ou Borderline. Entretanto, isto não foi confirmado pela avaliação psiquiátrica, apesar de a mesma ter recebido os diagnósticos de EDM e Transtorno de Personalidade Dependente.

Em relação ao fator N3, 50\% das pacientes apresentaram um valor acima do esperado, o que seria indicativo de Transtornos de Ansiedade, porém nenhuma paciente foi diagnosticada clinicamente com este tipo de Transtorno.

Em relação ao fator N4, 30\% das pacientes apresentaram um valor acima do normal, o que seria sugestivo de quadro depressivo. Destas, 15\% tiveram diagnóstico de EDM; $10 \%$ de EDM e Deficiência Mental Leve e 5\% de Transtorno Bipolar e Transtorno de Personalidade Borderline pela avaliação clínica.

Em relação à Tabela 4, podemos observar que no fator N1, o escore na $1^{\mathrm{a}}$ avaliação foi significativamente maior do que aquele da $2^{\mathrm{a}}$ e $3^{\mathrm{a}}$ avaliação (teste de Friedman: $\mathrm{P}=0,007$; com pós-teste de Student-Newman-Keuls: $\mathrm{P}<0,05)$. Nos fatores $\mathrm{N} 2, \mathrm{~N} 3$ e N4, não houve diferença significativa no escore da EFN entre os tempos analisados. Na soma dos escores padronizados, o escore na avaliação inicial foi significativamente maior do que os obtidos nas duas avaliações seguintes (teste de Friedman: $\mathrm{P}=0,005$; com pós-teste de Student-NewmanKeuls: $\mathrm{P}<0,05)$.

Observamos, ainda, de acordo com a Tabela 5, que a média dos escores percentílicos e gerais da EFN apresentou uma tendência à diminuição no decorrer do estudo, embora não tenha sido encontrada uma diferença estatisticamente significativa.

\section{Relação entre os resultados da EFN e SLEDAI nos tempos analisados}

Houve uma relação significativa entre a atividade da doença, avaliada de acordo com a pontuação na SLEDAI e o tempo de tratamento. Observamos que no decorrer do estudo houve uma diminuição da frequência de casos com atividade moderada/grave e um aumento dos casos de inatividade/atividade leve da doença.

Conforme exposto na Tabela 5, em todos os tempos analisados não houve relação significativa entre a pontuação na SLEDAI e o escore da EFN (teste do qui-quadrado, $1^{\text {a }}$ avaliação: $\mathrm{P}=0,088 ; 2^{\mathrm{a}}$ avaliação: $\mathrm{P}=0,348 ; 3^{\mathrm{a}}$ avaliação: $\mathrm{P}=0,371$ ). Não encontramos, portanto, uma relação entre alterações da personalidade e atividade do LES.

\section{DISCUSSÃO}

Verificamos que a maior parte das pacientes era afro-descendente, em concordância com vários trabalhos da revisão da 
literatura. ${ }^{14-17} \mathrm{~A}$ idade média foi semelhante à encontrada na maior parte dos estudos; ${ }^{14-17}$ porém, outros trabalhos ${ }^{41,23}$ obtiveram amostras com pacientes de maior faixa etária. A maior parte das pacientes declarou-se casada, como nos estudos de Segui et al..$^{18}$ e Dobkin et al. ${ }^{23}$

A média de escolaridade das participantes $(10,1 \pm 2,0$ anos) é inferior à referida em artigos internacionais. ${ }^{41,24}$ Em relação à ocupação/profissão, cerca de 55\% declararam realizar apenas atividades domésticas; estes dados diferem também da maioria dos estudos internacionais, como o de Segui et al. ${ }^{18}$ em que apenas $25 \%$ das pacientes declararamse donas-de-casa.

Verificamos que no decorrer do estudo houve uma diminuição da frequência de casos classificados pela EFN como normais e um aumento dos casos classificados como anormais. Acreditamos que isto se explique parcialmente pelo fato de que, na $2^{\mathrm{a}}$ avaliação, seis pacientes (46,1\%) diagnosticadas como deprimidas na avaliação inicial estavam tomando antidepressivo, e quatro $(30,7 \%)$ na $3^{\mathrm{a}}$ avaliação. O uso desta medicação pode ter diminuído as pontuações nas subescalas relacionadas à depressão e ansiedade. Entretanto, o esperado com o uso de antidepressivos seria uma normalização dos escores gerais, e não uma diminuição para níveis considerados abaixo do normal.

Observamos ainda que não houve uma relação significativa entre a avaliação psiquiátrica e o escore obtido na EFN. Não foi confirmado em nosso estudo, portanto, conforme dados da literatura, ${ }^{34}$ uma correlação da EFN com a nosologia psiquiátrica atual.

A prevalência de transtornos psiquiátricos em nossa amostra foi bastante alta $(75 \%)$.

Este índice se mostrou superior aos encontrados por Waterloo et al. ${ }^{41}$ (50\%) e Hutchinson et al. ${ }^{42}$ (44\%). Talvez esta diferença se explique pelo número restrito de pacientes em nossa amostra.

Verificamos através da literatura que a depressão é um diagnóstico significativamente relacionado ao LES. A prevalência de depressão em nossa $1^{\mathrm{a}}$ avaliação foi de $65 \%$, superior à encontrada por Waterloo et al..$^{41}(28 \%)$, Hutchinson et al. ${ }^{42}$ (27\%) e Nery et al. ${ }^{30}(23 \%)$. Mais uma vez, acreditamos que esta diferença se deve ao número de pacientes avaliadas.

Uma paciente $(5 \%)$ teve o diagnóstico de Transtorno de Personalidade Dependente. Não encontramos na literatura nenhuma referência a este diagnóstico em pacientes com LES. Entretanto, existem estudos ${ }^{41,23}$ que verificaram nas pacientes com LES aspectos psicológicos compatíveis com este transtorno, porém nestes trabalhos os autores não relataram este diagnóstico.

\section{Tabela 1}

Escores gerais da Escala Fatorial de Ajustamento Emocional/Neuroticismo (EFN), na avaliação inicial, em 3 e 6 meses; Campo Grande, 2004-2006

\begin{tabular}{lccc} 
Escore na EFN & $\begin{array}{c}\text { Inicial } \\
(\mathbf{n}=\mathbf{2 0})\end{array}$ & $\begin{array}{c}\mathbf{3} \text { meses } \\
(\mathbf{n}=\mathbf{1 9})\end{array}$ & $\begin{array}{c}\mathbf{6} \text { meses } \\
(\mathbf{n}=\mathbf{1 7})\end{array}$ \\
\hline & $\mathbf{n}^{\circ}(\%)$ & $\mathbf{n}^{\circ}(\%)$ & $\mathbf{n}^{\circ}(\%)$ \\
\hline Anormal (menos que 80 pontos) & $02(10 \%)$ & $05(26 \%)$ & $05(29 \%)$ \\
\hline Normal (80-120 pontos) & $17(85 \%)$ & $14(74 \%)$ & $11(65 \%)$ \\
\hline Anormal (mais que 120 pontos) & $01(05 \%)$ & $00(00 \%)$ & $01(06 \%)$ \\
\hline
\end{tabular}

\section{Tabela 2}

Diagnóstico psiquiátrico, de acordo com a avaliação psiquiátrica inicial, pelos critérios do CID 10, em relação ao escore na EFN, Campo Grande, 2004-2006

\begin{tabular}{lccc}
$\begin{array}{l}\text { Avaliação Psiquiátrica } \\
\text { (CID 10) }\end{array}$ & \multicolumn{2}{c}{ Escore na EFN } & Total \\
\hline Diagnóstico & $\begin{array}{c}\text { Normal } \\
\mathbf{n}^{\circ}(\%)\end{array}$ & $\begin{array}{c}\text { Anormal } \\
\mathbf{n}^{\circ}(\%)\end{array}$ & $\mathbf{N}^{\circ}(\%)$ \\
\hline $\begin{array}{l}\text { Episódio Depressivo } \\
\text { Moderado (EDM) }\end{array}$ & $10(50 \%)$ & - & $10(50 \%)$ \\
$\begin{array}{l}\text { EDM e Transtorno de } \\
\text { Personalidade Dependente }\end{array}$ & - & $01(05 \%)$ & $01(05 \%)$ \\
\hline $\begin{array}{l}\text { EDM e Deficiência Mental Leve } \\
\text { Transtorno Bipolar e Transtorno } \\
\text { de Personalidade Borderline }\end{array}$ & $02(10 \%)$ & - & $02(10 \%)$ \\
\hline $\begin{array}{l}\text { Uso abusivo de álcool e } \\
\text { dependência de tabaco }\end{array}$ & $01(05 \%)$ & - & $01(05 \%)$ \\
\hline $\begin{array}{l}\text { Sem diagnóstico psiquiátrico } \\
\text { Total }\end{array}$ & $03(15 \%)$ & $02(10 \%)$ & $05(25 \%)$ \\
\hline
\end{tabular}

CID 10: Classificação Internacional de Doenças da Organização Mundial de Saúde - $10^{\mathrm{a}}$ revisão; EFN: Escala Fatorial de Ajustamento Emocional/Neuroticismo.

\section{Tabela 3}

Número de pacientes e pontuação dos escores percentílicos das subescalas da EFN, na avaliação inicial, Campo Grande, 2004-2006

\begin{tabular}{lcccc}
\hline & \multicolumn{3}{c}{ Escores percentílicos da EFN } & \\
\cline { 2 - 4 } Fatores & Menos que & Entre 30 e & Mais que & \multirow{2}{*}{ Total } \\
\hline da EFN & $\mathbf{3 0 \text { pontos }}$ & $\mathbf{7 0}$ pontos & $\mathbf{7 0 \text { pontos }}$ & \\
\hline N1 & $\mathbf{n}^{\circ}(\%)$ & $\mathbf{n}^{\circ}(\%)$ & $\mathbf{n}^{\circ}(\%)$ & $\mathbf{n}^{\circ}(\%)$ \\
N2 & $03(15 \%)$ & $09(45 \%)$ & $08(40 \%)$ & $20(100 \%)$ \\
N3 & $06(30 \%)$ & $13(65 \%)$ & $01(05 \%)$ & $20(100 \%)$ \\
N4 & $03(15 \%)$ & $07(35 \%)$ & $10(50 \%)$ & $20(100 \%)$ \\
\hline
\end{tabular}

EFN: Escala Fatorial de Ajustamento Emocional/Neuroticismo; N1: Subescala de Vulnerabilidade; N2: Subescala de Desajuste Psicossocial; N3: Subescala de Ansiedade; N4: Subescala de Depressão. 
Tabela 4

Resultados dos escores percentílicos para cada fator e soma dos escores padronizados da EFN, na avaliação inicial, em 3 e 6 meses, Campo Grande, 2004-2006

\begin{tabular}{|c|c|c|c|c|c|}
\hline \multirow[b]{2}{*}{ Tempos analisados } & \multicolumn{4}{|c|}{ Escores percentílicos por fator } & \multirow[b]{2}{*}{$\begin{array}{c}\text { Soma dos escores } \\
\text { padronizados }\end{array}$} \\
\hline & N1 & N2 & N3 & N4 & \\
\hline Inicial & $\begin{array}{c}59,47 \\
+31,55^{*}\end{array}$ & $\begin{array}{c}39,63 \\
\pm 26,66\end{array}$ & $\begin{array}{r}64,55 \\
\pm 28,34\end{array}$ & $\begin{array}{c}53,59 \\
\pm 30,93\end{array}$ & $\begin{array}{r}102,03 \\
\pm 13,58\end{array}$ \\
\hline 3 meses & $\begin{array}{c}47,27 \\
\pm 36,09\end{array}$ & $\begin{array}{c}29,34 \\
\pm 23,74\end{array}$ & $\begin{array}{c}51,84 \\
\pm 31,22\end{array}$ & $\begin{array}{r}38,92 \\
\pm 31,19\end{array}$ & $\begin{array}{c}95,19 \\
\pm 14,29\end{array}$ \\
\hline 6 meses & $\begin{array}{c}42,90 \\
\pm 34,61\end{array}$ & $\begin{array}{c}23,82 \\
\pm 29,18\end{array}$ & $\begin{array}{c}45,14 \\
\pm 38,00\end{array}$ & $\begin{array}{c}39,38 \\
\pm 30,20\end{array}$ & $\begin{array}{r}92,05 \\
\pm 17,46\end{array}$ \\
\hline Valor de P & $0,007^{* *}$ & 0,126 & 0,054 & 0,112 & $0,005^{* *}$ \\
\hline
\end{tabular}

* Média \pm desvio padrão da média

${ }^{\star}$ Teste de Friedman com pós-teste de Student-Newman-Keuls $(P<0,05)$, escore no Tempo 0 significativamente maior que nos Tempos 1 e 2.

EFN: Escala Fatorial de Ajustamento Emocional/Neuroticismo; N1: Subescala de Vulnerabilidade; N2: Subescala de Desajuste Psicossocial; N3: Subescala de Ansiedade; N4: Subescala de Depressão.

\section{Tabela 5}

Atividade da doença de acordo com a pontuação na SLEDAI, em relação à pontuação na EFN, na avaliação inicial, em 3 e 6 meses, Campo Grande, 2004-2006

\begin{tabular}{|c|c|c|c|c|c|c|}
\hline \multirow{3}{*}{ Pontuação na SLEDAI } & \multicolumn{6}{|c|}{ Pacientes de acordo com o escore na EFN } \\
\hline & \multicolumn{2}{|c|}{$\begin{array}{c}\text { Inicial } \\
(\mathbf{n}=20)\end{array}$} & \multicolumn{2}{|c|}{$\begin{array}{l}3 \text { meses } \\
(n=19)\end{array}$} & \multicolumn{2}{|c|}{$\begin{array}{l}6 \text { meses } \\
(n=17)\end{array}$} \\
\hline & Normal & Anormal & Normal & Anormal & Normal & Anormal \\
\hline Atividade da doença & \multicolumn{2}{|c|}{$n^{\circ}(\%)$} & \multicolumn{2}{|c|}{$n^{\circ}(\%)$} & \multicolumn{2}{|c|}{$N^{\circ}(\%)$} \\
\hline Inatividade ( 0 pontos $)$ & $00(00 \%)$ & $01(05 \%)$ & $02(11 \%)$ & $00(00 \%)$ & $02(12 \%)$ & $00(00 \%)$ \\
\hline Atividade leve (1-3 pontos) & $02(10 \%)$ & $00(00 \%)$ & $01(05 \%)$ & $01(05 \%)$ & $01(06 \%)$ & $02(12 \%)$ \\
\hline Atividade moderada (4-7 pontos) & $04(20 \%)$ & $01(05 \%)$ & $07(37 \%)$ & $04(21 \%)$ & $02(12 \%)$ & $02(12 \%)$ \\
\hline Atividade grave (8 pontos ou mais) & $11(55 \%)$ & $01(05 \%)$ & $04(21 \%)$ & $00(00 \%)$ & $06(35 \%)$ & $02(12 \%)$ \\
\hline Valor de $\mathrm{P}$ (teste do qui-quadrado) & \multicolumn{2}{|c|}{0,088} & \multicolumn{2}{|c|}{0,348} & \multicolumn{2}{|c|}{0,371} \\
\hline
\end{tabular}

SLEDAl: Systemic Lupus Erythematosus Disease Activity Index; EFN: Escala Fatorial de Ajustamento Emocional/Neuroticismo.

Uma paciente (5\%) teve o diagnóstico de Transtorno Bipolar e Transtorno de Personalidade Borderline. Novamente, não encontramos na literatura nenhuma referência a este último transtorno em pacientes com LES; porém, também existem trabalhos que encontraram nas pacientes lúpicas sintomas típicos de Transtorno de Personalidade Borderline, ${ }^{41,23,43}$ mas esses autores também não referiram este diagnóstico em suas avaliações.

Observamos que a média dos escores percentílicos e gerais da EFN teve uma tendência à diminuição no decorrer do estudo, embora não tenha sido encontrada uma diferença estatisticamente significativa. Como já foi relatado, acreditamos que a diminuição dos escores pode estar relacionada ao uso de antidepressivos por algumas pacientes na $2^{\mathrm{a}}$ e $3^{\mathrm{a}}$ avaliação.
Entretanto, nem todas as pacientes deprimidas aderiram à medicação prescrita pela psiquiatria.

Verificamos, portanto, que os escores da EFN, como os próprios autores ${ }^{34}$ afirmam, são apenas indicativos de Transtornos de Personalidade. Apenas uma paciente (5\%) teve diagnóstico clínico de Transtorno de Personalidade Dependente confirmado pelos escores geral e específico (N1) da escala. Entretanto, apesar de apresentar um diagnóstico de depressão (EDM), seu escore percentílico relacionado a este fator (N4) estava dentro do normal. Entretanto, a outra paciente que também recebeu o diagnóstico de Transtorno de Personalidade (Borderline) pela avaliação clínica obteve valores normais nos escores gerais da EFN e, paradoxalmente, um valor baixo (5 pontos) na subescala relacionada a este fator (N2). 
Ainda quanto aos escores percentílicos das subescalas, como podemos observar na Tabela 3 , um número substancial da amostra apresentava alterações compatíveis com Transtornos de Personalidade. Entretanto, apenas duas pacientes (10\%) receberam estes diagnósticos de acordo com a avaliação clínica psiquiátrica, que ainda é considerada padrão-ouro de certeza diagnóstica. Segundo Bernik, ${ }^{43}$ mesmo antes do surgimento de escalas de avaliação, a observação cuidadosa sempre foi a mais valiosa fonte de informação dos fenômenos psiquiátricos. Entretanto, devemos salientar que, apesar de nossa avaliação psiquiátrica estar embasada nos critérios do CID $10,{ }^{36}$ ainda é bastante subjetiva, sendo a escala um método mais objetivo de avaliação.

Nossos achados coincidem ainda com os de Vertzman \& Pinheiro, apud Lemle (2005), ${ }^{29}$ que mesmo usando outro referencial (psicanalítico), encontraram, como em nosso estudo, pacientes com os mais variados tipos de organização psíquica, o que contraria a proposta de que as pacientes lúpicas teriam um modelo próprio, homogêneo e específico de funcionamento psicológico.

Em virtude do número reduzido de nossa amostra, não podemos generalizar os resultados para toda a população de pacientes com LES; entretanto, observamos, através da análise dos prontuários, que a maioria das pacientes que tiveram melhor evolução no transcorrer da pesquisa foram as que tiveram melhor aderência tanto ao tratamento reumatológico como ao psiquiátrico. De acordo com Fráguas Jr. ${ }^{44} \mathrm{o}$ tratamento eficaz da depressão melhora o quadro clínico de base e a qualidade de vida do paciente, reduzindo a utilização inadequada dos serviços médicos.

Não encontramos neste estudo uma relação entre alterações da personalidade e atividade do LES. Estes dados são semelhantes aos de Ishikura et al., ${ }^{22}$ que também não encontraram relação entre as características psicológicas de suas pacientes e a atividade do LES. Porém, são diferentes dos achados de Segui et al..${ }^{18}$ Ward et al. ${ }^{24}$ e Yuko et al..${ }^{25}$ que encontraram relação entre sintomas psiquiátricos (incluindo alterações de personalidade) e a atividade da doença.

Concluindo, verificamos em nosso estudo uma prevalência importante de transtornos psiquiátricos (75\%), mas não de Transtornos de Personalidade (apenas 10\%). O diagnóstico mais prevalente foi o de Episódio Depressivo Moderado (EDM), em $65 \%$ dos casos. Não foi encontrado, em nossa amostra, um padrão típico de personalidade ou prevalência específica de um Transtorno de Personalidade.

Não foi encontrada relação significativa entre o diagnóstico psiquiátrico (pelos critérios da CID 10) na $1^{\mathrm{a}}$ avaliação e os escores geral e percentílico da EFN. Em todos os tempos analisados não houve também relação significativa entre a pontuação na SLEDAI e o escore da EFN; não foi verificada, portanto, associação entre alterações da personalidade e atividade da doença. De acordo com as probabilidades estatísticas, se tivéssemos uma amostra maior, talvez várias associações entre aspectos do LES e quadros psiquiátricos, que não foram confirmadas neste trabalho, apresentariam significância estatística. Fazem-se necessários, portanto, estudos com um número maior de pacientes para que estas hipóteses sejam mais acuradamente verificadas.

\section{AGRADECIMENTOS}

Às pacientes com Lúpus Eritematoso Sistêmico que se dispuseram a participar da pesquisa, por sua confiança. A todos os colegas que colaboraram voluntariamente em todas as fases de realização deste trabalho, por sua extrema generosidade. 


\section{REFERÊNCIAS}

\section{REFERENCES}

1. Borba EF, Latorre LC, Brenol JCT et al. Consenso de Lúpus Eritematoso Sistêmico. Rev Bras Reumatol 2008; 48(4):196-207.

2. Moreira MD, Mello Filho J. Psicoimunologia hoje. In: Mello Filho J. Psicossomática hoje. Porto Alegre: Artmed 1992, pp. 119-51.

3. Bonfá ESDO, Borba Neto EFB. Lúpus Eritematoso Sistêmico. In: Bonfá ESDO, Ioshinari NH: Reumatologia para o clínico. São Paulo: Roca 2000, pp. 25-33.

4. Hahn BH. Pathogenisis of Systemic Lupus Erythematosus. In: Kelley WN, Harris Jr. ED, Ruddy S, Sledge CB. Textbook of Rheumatology. v. 2, 5 ed. Philadelphia: W.B. Saunders Co, 1997, pp. 1089-103.

5. Araújo GRB. De "lupus" et homine: contribuições a um espaço de atuação do psiquiatra em Hospital Geral. (Dissertação de Mestrado). Rio de Janeiro: Universidade Federal do Rio de Janeiro, 1989.

6. Miguel Filho EC. Alterações Psicopatológicas no Lúpus Eritematoso Sistêmico. (Tese de Doutorado). São Paulo: Universidade de São Paulo, 1992.

7. Moran M, Dubester S. Connective Tissue diseases. In: Stoudemire A, Fogel BS: Psychiatric Care of the Medical Patient. New York: Oxford University Press, 1993. pp. 739-745.

8. Leritz E, Brandt J, Minor M, Reis-Jensen F, Petri, M. Neuropsychological functioning and its relationship to antiphospolipid antibodies in patients with Systemic Lupus Erythematosus. J Clin Exp Neurops 2002; 24:527-33.

9. Kelly MJ, Rogers, MP. Neuropsychiatric Aspects of Systemic Lupus Erythemathosus. In: Stoudemire A, Fogel B. Medical-Psychiatric Practice. v. 3. Washington DC: American Psychiatric Press, 1995. pp. 183-214.

10. Maes MBE, Bonaccorso S, Hunsel FV et al. Increased 24-hour urinary cortisol excretion in patients with post-traumatic stress disorder and patients with major depression, but not in patients with fibromyalgia. Acta Psych Scand 1998; 98:328-35.

11. Ayache DCG, Costa IP. Alterações da personalidade no Lúpus Eritematoso Sistêmico. Rev Bras Reumatol 2005; 45(5):313-8.

12. Liang MH, Rogers M, Larson M et al. The psychosocial impact of Systemic Lupus Erythematosus and Rheumatoid Arthritis. Arthritis Rheum 1984; 27:13-9.

13. Cabral MA, Giglio JS, Stangehaus G. Características de personalidade de pacientes artríticos reumatóides, tratados no ambulatório de um Hospital-Escola. Revista ABP-APAL 1986; 8:102-106.

14. Liang MH, Socher SA, Larson MG, Schur PH. Reliability and validity of six systems for the clinical assesment activity in Systemic Lupus Erithematosus. Arthritis Rheum, 1989; 32(9):1107-18.

15. Lim LC, Lee T, Boey, M. Psychiatric manifestation of Systemic Lupus Erythematosus in Singapore: A cross - culture comparison. Br J Psychiatry 1991; 159:520-3.

16. Braden CJ. Patterns of change over time in learned response to chronic illness among participants in a Systemic Lupus Erythematosus selfhelp course. Arthritis Care Res 1991; 4(4):158-67.

17. Onda K, Kato SA. Clinical study psychopathology in Systemic Lupus Erythematosus. Seishin Shinkeigaku Zasshi 2000; 102(7):616-39.

18. Segui J, Ramos-Casals M, Garcia-Carrasco et al. Psychiatric and psychosocial disorders in patients with Systemic Lupus Erythematosus: a longitudinal study of active and inactive stages of the disease. Lupus 2000; 9(8):584-8.

19. Sato EI. Manifestações psiquiátricas da corticoterapia. In: Psiquiatria na prática médica.[periódico na internet]. Acessado em 23/10/2004. [aproximadamente 2 p.] Disponível em http://www.unifesp.br/dpsiq/ polbr/ppm/atu4_01.htm.

20. Santoantonio J. Estudo de características da personalidade de adolescentes com Lúpus Eritematoso Sistêmico por meio do método de Rorschach. (Tese de Doutorado). São Paulo, Universidade Federal de São Paulo (UNIFESP), 2001.

21. Bae SC, Hashimoto H, Karlson, EW, Liang MH, Daltroy L H. Variable effects of social support by race, economic status, and disease activity in Systemic Lupus Erythematosus. J Rheumatol 2001; 28(6):1245-51.

22. Ishikura $\mathrm{R}$, Morimoto $\mathrm{N}$, Tanaka $\mathrm{K}$ et al. Factors associated with anxiety, depression and suicide ideation in female outpatients whit SLE in Japan. Clin Rheumatol 2001; 20(6):394-400.

23. Dobkin P L, Costa D, Fortin PR et al. Living with lupus: a prospective pan-Canadian study. J Rheumatol 2001; 28(11):2442-8.

24. Ward MM, Marx AS, Barry NN. Psychological distress and changes in the activity of Systemic Lupus Erythematosus. Rheumatology (Oxford) 2002; 41(2):184-8.

25. Yuko M, Takeshi S, Yumiko A, Toru H, Shigeru H. Psychological profiles and health status in Japanese female patients with Systemic Lupus Erythematosus: the Miyagi Lupus Collaborative Study. J Epidemiol 2002; 2(2):55-3.

26. Martins JM, Alves J, Trinca A et al. Personality, brain asymmetry, and neuroendocrine reactivity in two immune-mediate disorders: a preliminary report. Brain Behav Immun 2002;16:383-97.

27. Trysberg T, Tarkowsky A. Cerebral inflammation and degeneration in Systemic Lupus Erythemathosus. Curr Opin Rheumatol 2004; 16:527-33.

28. Nery FG, Borba EF, Lotufo Neto, FL. Influência do estresse psicossocial no Lúpus Eritematoso Sistêmico. Rev Bras Reumatol 2004; 44(5):355-61. 
29. Lemle, M. Lúpus e patologias narcísicas: existe relação? Boletim on-line FAPERJ [periódico na internet]. Acessado em 27/05/2006. [aproximadamente 2 p.]. Disponível em http://www.faperj.br/ boletim_interna.phtml?obj_id=1974\#topo.

30. Nery, FG, Borba EF, Hatch JP, Soares JC, Bonfa E, Neto FL. Major depressive disorder and disease activity in Systemic Lupus Erythematosus. Compr Psychiatry 2007; 48(1):14-9.

31. Nery FG, Borba EF, Viana VS et al. Prevalence of depressive and anxiety disorders in Systemic Lupus Erythematosus and their association with anti-ribosomal P antibodies. Prog Neuropsychopharmacol Biol Psychiatry. 2008; 32(3):695-700.

32. Tan EM, Cohen AS, Fries JF et al. Special article: The 1982 revised criteria for the classification of Systemic Lupus Erythematosus. Arthritis Rheum 1982; 25:1271-7.

33. Almeida OP. Instrumentos para avaliação de pacientes com demência. In: Gorenstein C, Andrade LHSG, Zuardi AW, eds. Escalas de Avaliação Clínica em Psiquiatria e Psicofarmacologia. São Paulo: Lemos, 2000. pp. 331-5.

34. Hutz CS, Nunes CHSS. Escala Fatorial de Ajustamento Emocional/ Neuroticismo (E F N). São Paulo: Casa do Psicólogo, 2001.

35. American Psychiatric Association. Diagnostic and statistical manual of mental disorders (4 ${ }^{\text {th }}$ ed). Washington, DC: APA, 1994.

36. Organização Mundial de Saúde-OMS: Classificação de Transtornos Mentais e de Comportamento. Porto Alegre: Artes Médicas, 1993. pp. 194-206.
37. Hutz, C.S.(Instituto de Psicologia da UFRGS) Comunicação pessoal. Porto Alegre, 2006. Recebida por rdayache@terra.com. br, em 2/5/2006.

38. Bombardier C, Gladman, D D, Urowitz M B, Caron D, Chang, C $\mathrm{H} \&$ Committee on Prognosis Studies in SLE. Derivation of the SLEDAI - A disease activity index for lupus patients. Arthritis Rheum 1992; 35:630-640.

39. Cook RJ, Gladman DD, Pericak D, Urowitz MB. Prediction of short-term mortality in Systemic Lupus Erythematosus with time dependent measures of disease activity. J Rheumatol 2000; 27(8):1892-5.

40. Shott S. Statistics for health professionals. London: W.B. Saunders Company, 1990.

41. Waterloo K, Omdal R, Husby G, Mellgren, S. Emotional status in Systemic Lupus Erythematosus. Scand J Rheumatol 1998; 27:410-4.

42. Hutchinson GA, Nehall FRC, Simeon DT. Psychiatric Disorders in Systemic Lupus Erythematosus. W I Med J 1996; $45: 48$.

43. Bernik MA. Dificuldades na utilização de escalas de avaliação de sintomas ansiosos em psicofarmacologia clínica e experimental. Rev Psiq Clin 25(6) Edição Especial: 326-30, 1998. Acessado em 23/5/2006. [aproximadamente 6 p.]. Disponível em http://www. hcnet.usp.br/ipq/revista/r256/ansi256h.htm.

44. Fráguas Jr R: Depressão no contexto médico. In: Lafer B, Almeida OP, Fráguas Jr R, Miguel EC, eds. Depressão no ciclo da vida. São Paulo: Editora ArtMed; 2000. pp. 179-186. 